\title{
Reducing Handoff Delay In WLan Using Selective Proactive Context CACHING
}

\author{
Teck Chaw Ling ${ }^{1}$, Jen Funn Lee ${ }^{1}$, Kian Pee Hoh ${ }^{2}$ \\ ${ }^{1}$ Faculty of Computer Science and Information Technology, \\ University of Malaya, 50603 Kuala Lumpur, Malaysia. \\ ${ }^{2}$ School of Electronics \& Info-Comm Technology, \\ ITE College East, Singapore 486047 \\ E-mail: tchaw@um.edu.my, 1jf81@yahoo.com,hoh_kian_pee@ite.edu.sg
}

\begin{abstract}
In IEEE 802.11 wireless networks, continuous connectivity which allows user mobility and maintains network utilization is one of the most important requirements. In order to establish and keep service state information during the handoff process, the context transfer protocol is used to transfer service state from the originally associated base station to another base station. These base stations exchange messages to establish a secure channel between the APs for transmitting the STA context information. The entire process, however, inevitably increases the overall handoff latency. To resolve the predicament, we propose the Selective Proactive Context Caching (SPCC) technique to proactively propagate security context of the mobile client to a selected set of neighboring base stations before re-association occurs. Unlike the conventional 802.11 handoff mechanism in which cooperation among neighboring base stations does not exist, a selected neighboring base stations in SPCC exchange link quality information of the mobile client through LAN and this information is stored into cache memory. Based on the link quality information, the originally associated AP selects a set of neighboring APs and forwards the security context of mobile station through the LAN. Simulation results show an improvement of $62 \%$ in reducing the re-association handoff delay. Specifically, the re-association delay is reduced from $3.2 \mathrm{~ms}$ in the 802.11 handoff mechanism to $1.2 \mathrm{~ms}$ when SPCC is applied.
\end{abstract}

\section{Keywords: Handoff Delay, Context Caching, Re-association, Selective Proactive Context Caching}

\section{INTRODUCTION}

Wireless-Fidelity (Wi-Fi), a wireless technology based on the IEEE 802.11 standards has proliferated widely across the world. Wi-Fi has changed the conventional wired way of accessing network resources. It is now being widely deployed in home-based LANs, office-based LANs and publicly available hot spots, which are areas around a central antenna where people can gain access to the Internet with a laptop equipped with a wireless card. Wireless access allows wireless clients to be mobile compared to wired connection

The use of multimedia and real-time applications such as video conferencing, IP telephony and interactive online games via Wi-Fi connections is also growing rapidly. The performance of these applications are highly dependent on the performance of wireless connectivity especially when the tendency of moving is high. Seamless connection is desired in order to avoid unpredictable packet loss, delay and jitter when the wireless client moves out of its original radio range to a radio range covered by another base station. Thus, wireless handoff and mobility management have been scrutinized and studied by network researchers.

In addition, the security features used in wireless networks indirectly increase the overall handoff latency. The protocol involved in the secure transfer of station service state information from the originally associated Access Point (AP) to the newly selected AP further increases handoff latency due to re-association. The selections of APs to which the service state of station will be transferred generally do not consider whether the station has visited the radio range covered by the specific AP. Therefore, bandwidth and memory may be wasted in order to transfer the service state of station and to store that information.

In this paper, we examine all of these issues and focus on how to minimize the Layer 2 re-association latency. Our solution, called Selective Proactive Context Caching (SPCC), reduces the re-association delay in the standard handoff (with Inter Access Protocol Point (IAPP)) by predicting the next possible APs where a station is likely to connect. We have implemented the SPCC algorithm using the OMNeT++ simulator equipped with INET 
Framework and showed that SPCC is able to reduce the re-association delay as compared to the regular handoff with IAPP.

The rest of the paper is organized as follows. In Section 2 we review the regular 802.11 handoff mechanisms and previous works in reducing handoff delays. Next, Section 3 describes the SPCC algorithm in detail, as well as its advantages. Section 4 discusses analysis of our simulation implementation. Finally, our results are summarized and possible future work is discussed in Section 5.

\section{BACKGROUND AND RELATED WORK}

This section first gives a preliminary background of the IEEE 802.11 handoff procedure. Next, the functionalities of IAPP are described, followed by previous works related to reducing handoff delays.

\subsection{Background}

The IEEE 802.11 WLANs Layer 2 handoff procedures can be divided into three main phases: (i) Discovery (ii) Reauthentication and (iii) Re-association. Figure 1 shows the sequence of message exchanged between the STA and the AP during handoff.

The discovery phase starts immediately after handoff is initiated. The mobile Station (STA) may not be able to communicate with its associated AP. In order to find the potential APs within its radio range, the STA tunes into each predefined channels and listens for beacon frames which are sent out periodically (10ms by default) by the APs. From the beacon frames, the STA creates a candidate list of APs and decides with which AP to associate based on the received signal strength.

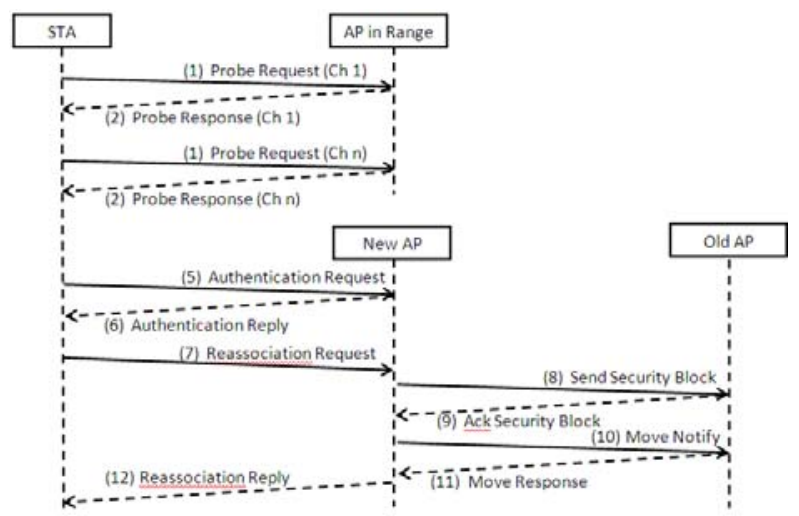

Fig 1: The handoff procedure as specified in IEEE 802.11 and IEEE 802.11f.

An STA shall operate in either a Passive Scanning or an Active Scanning mode. In Passive Scanning mode, the STA tunes into each channel and passively scans for any beacon frame for a duration no longer than a maximum duration defined by the ChannelTime parameter. In Active Scanning mode, the STA tunes into each channel and generates a Probe Request frame (step 1) containing the broadcast Basic Service Set Identification (BSSID) and broadcasts it to the APs in range. Messages 1 and 2 in Figure 1 show the active scanning steps. In the Active Scanning mode, there are four important parameters, namely ProbeDelay, ProbeTimer, MinChannelTime and MaxChannelTime. These parameters are defined by manufacturers and therefore are proprietary. ProbeDelay is the time taken by the Network Interface Card (NIC) to passively monitor the channel for activities before sending a Probe Request [10]. Immediately after a Probe Request is sent, the existing ProbeTimer is cleared and restarted. If no Probe Response frame is received when ProbeTimer reaches MinChannelTime, the STA declares that the channel is empty or no AP is in range and proceeds to the next channel. Otherwise, the STA waits until ProbeTimer reaches MaxChannelTime while collecting other potential Probe Response frames from other APs on the channel. The STA then processes all received Probe Response frames (step 2) and scans the next channel. Once every channel has been scanned, the STA selects the best AP for authentication and association based on the measured signal-to-noise ratio (SNR) from the AP list.

The re-authentication process involves re-authentication and re-association to the selected AP. The re-authentication phase is used by the STA to establish its identity with the AP that it wishes to communicate with. IEEE 802.11 
supports several authentication schemes such as Open System authentication, Shared Key authentication, WPA/WPA2 authentication and 802.1X Port-based Network Access Control. During this phase, the STA sends a reauthentication frame (step 5) to the selected AP and receives the re-authentication frame (step 6) as acknowledgement. Once the re-authentication is completed, the re-association process starts in order to enable the existing established association to be transferred from the old AP to the newly selected AP. The STA sends an 802.11 Re-association Request frame (step 7) to the AP and receives a re-association response (step 12) from the AP. During re-association, the APs involved also exchange station context information via the use of IAPP. [5]

The IAPP, which addresses the issue of interoperability among APs from multiple vendors, plays an important role during the handoff process. The IAPP ensures that an STA maintains only a single association with the wireless network at a specific time and is used for the secure transfer of state and context information between APs involved in a re-association process [8]. The context of a mobile station consists of the association states, timestamp, sequence number, BSSID, capability, security information, QoS, header compression, and AAA information [5][8].

The association and re-association phases change an STA's point of connectivity to the network. When an STA first associates to an AP, the AP broadcasts an Add-Notify packet to all other APs, triggering the STA's current association. Upon receiving an Add-Notify packet, the other APs clear all stale associations and state for the specific station to enforce a single point of association for the station. During the re-association process, the new AP can optionally send a Security Block packet (step 8) to the old AP in order to establish a secure communication channel for transferring the station context information and receive an Ack-Security-Block packet (step 9) as acknowledgement. Once the secure communication channel is established, the new AP sends a Move-Notify packet (step 10) to the old AP to request for the station context information. Then, the old AP replies with a MoveResponse packet (step 11) for acknowledgement. The use of Security Block and Ack-Security Block packets to establish a secure communication channel during re-association further increases the re-association latency. Although the IAPP allows for the secure communications between APs, it has increased the overall handoff latency because of its reactive nature.

The overall handoff latency in IEEE 802.11 WLANs can be divided into three delays: (i) Probe delay (ii) Reauthentication delay and (iii) Re-association delay. Probe delay covers the duration from the first Probe Request frame sent by the STA on the first channel to the time the STA receives the last Probe Response frame on the last channel during the scanning phase. Once the STA initiates a handoff, it must identify the set of potential candidate APs by scanning each channel. Thus, the latency of active scanning approach is equivalent to:

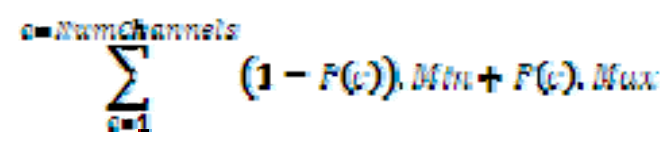

where $P(c)$ is the probability of one or more APs operating in channel c, Min represents the MinChannelTime and Max represents the MaxChannelTime [10].

Re-authentication delay is the latency incurred during the exchange of authentication frames between an STA and an AP as indicated by messages 5 to 6 in Figure 1. Authentication delay may vary depending on the authentication method used by the AP where two or four authentication frames are required during this process. Re-association delay is the latency incurred during the exchange of re-association frames between an STA and an AP as indicated by messages 7 and 12 in Figure 1. If IAPP is applied, the re-association delay is further increased: four additional messages (messages 8 through 11) are exchanged between the old AP and the new AP in order to securely transfer the STA's context information from the old AP to the new AP.

\subsection{Related Work}

There have been several previous attempts to reduce 802.11 handoff delays. Arunesh et al. [6] have showed that the probe delay contributes $90 \%$ to the total handoff latency and the latency varies depending on the environment, hardware capabilities and parameter selection. To reduce the probe delay, Kim et al. [7] proposed a selective channel scanning method using neighbor graph (NG) approach. This technique, first introduced by Arunesh et al. [8], uses the topological information on APs to reduce the number of channels to be scanned. A more aggressive approach to reduce probe delays is proposed by Vladimir Brik et al. [11] who use two radio interfaces to perform active scanning and data communication simultaneously. The primary interface is used for data transmission while the secondary is used for probing neighboring APs. The roles of both interfaces are swapped once the secondary interface has completed its association process. 
Kishore Ramachandran et al. [16] proposed a similar approach called the make-before-break algorithm in which the STA uses two radio cards to gather neighboring information through periodic probing. Similarly, the functions of the two radio cards are switched for data communication and probing. Jaeouk OK et al. [18] extended the two radio card concept in their attempt to eliminate the probe delay and proposed an approach called Shared Beacon Channel. An STA is equipped with two radio cards, one for data communication and another one for listening to a shared beacon channel to determine the next AP based on its handoff policy. The feasibility of using two radio receivers remains an issue to be questioned in terms of level of interference, power consumption and cost of development.

Ishwar Ramani et al. [10] proposed a more practical handoff mechanism called SyncScan that makes use of scanning time synchronization. This mechanism uses a special type of beacon frame to be periodically broadcast by the APs in order to identify themselves to the STAs in range and to synchronize the service state information with all the currently associated STAs. The timing of beacon broadcast on each channel is synchronized between the STAs and the APs so that the STAs can efficiently identify all the neighboring APs by regularly switching to each channel; this method minimizes the time it losses communication with its own AP.

Vivek Mhatre et al. [17] propose a similar approach that uses the concept of continuous monitoring of neighboring APs. In this approach, the STA driver continuously monitors the link quality of all the APs operating on the STA's current channel, as well as all the neighboring APs operating on its overlapping channel through beacon signal strength measurement. The driver source code is modified to monitor APs operating on overlapping channels. Extending the work of Vivek [17], Wu et al. [19] presented another trigger method using Proactive Scan. The key idea of this work is to decouple the time consuming channel scanning from the actual handoff, and to initiate the scanning process early and in a way that allows ongoing traffic. Additionally, Proactive Scan considers both uplink and downlink quality before handoff is triggered.

The re-authentication process is also one of the delays that constitute the overall handoff latency. Previous works on reducing the re-authentication delay are based on different types of authentication models. Pack et al. [2] proposed a fast inter-AP handoff scheme in which the STA performs the authentication process not only for its own associated AP but also for the neighboring APs based on IEEE 802.1x model when handoff is initiated. A mechanism called Frequent Handoff Region (FHR) is used to determine the set of adjacent APs by monitoring the APs' locations and users' movements. The FHR keeps record of APs with which the STAs are likely to connect next. To further reduce the re-authentication delay and to provide better security, Aura et al. [14] presented a protocol that involves the use of previous credential in the re-authentication process. During handoff, the STA receives a credential from its old AP, which it has successfully authenticated. This credential is proof of its verified honest behaviour (previous successful authentication). When the STA attempts to link up with the new AP, it presents this credential to the new AP. Pack et al. [21] proposed a selective neighboring cache scheme, which propagates the STA's context to some selected neighbor APs. In this method, each neighbor AP is assigned with a different weight to the NG. Using this method, a constant update of the weight table is necessary and increases the overall overhead.

Previous analysis on IEEE 802.11 MAC layer handoff by Arunesh et al. [6] shows that re-association delay contributes $15.37 \mathrm{~ms}$ to the overall handoff delay. To reduce the re-association delay when IAPP is applied, Arunesh et al. [8] proposed an approach called context caching by using neighbour graph (NG). Their major contribution is to propagate the STA security contexts proactively to the next potential APs in order to eliminate the IAPP latency during re-association.

Previous works that focus on reducing the overall handoff delay are generally based on context transfer mechanism. The key objective of context transfer is to establish and keep service state information which is essential to process and forward packets in a way that suits specific service requirement. Ha et al. [15] used the idea of context transfer and proposed a proactive scheme to estimate the best time to transfer context information associated with the STA. In this approach, the handoff is triggered at a planned time to avoid unnecessary handoff.

Wang \& Bao [12] used the same concept and presented an approach called Mobile AP to determine the best time to trigger the handoff. In this approach, the handoff decision is made by the AP instead of by the STA. Each AP collects the STA-AP network connection quality information and reports the Received Signal Strength Indicator (RSSI) information from all overheard STA data frames. Then the AP selectively broadcast its local STA-AP connection and link quality information of both the associated and not associated STAs. A handoff decision is made by an AP if another AP reports the best link quality among all the APs. Once the old AP initiates handoff, the old AP transfers the STA-AP context information to the new AP using Mobile AP protocol (similar to IAPP). 
The mobility management is one of the major concerns in IEEE 802.11 WLANs where the handoff mechanism affects the performance of multimedia applications. To reduce the total handoff latency, some approaches have been proposed, such as handoff triggering techniques, proactive scanning mechanism and context transfer. Nevertheless, the efficiency and overhead in the existing techniques are issues that can be further improved. Context transfer [8] [12] is one of the existing techniques to transfer the old association states between the STA and the old AP to the new AP. The timing of context transfer and the prediction mechanism to identify the next possible APs to which the STA will connect next are the major problems in this approach. Inaccuracy of the timing and the prediction mechanism may lead to more overheads and resources wastage where context information is transferred to non-related APs. In addition, the exchange of STA-AP link quality information approach to identify next possible AP introduces regular overhead as the APs broadcast link quality information continuously [12]. Therefore, there is a need to develop a proactive context caching technique to further reduce the re-association delay and overheads as well as to minimize resources wastage.

\section{SPCC}

This section presents our proposed SPCC mechanism and the algorithm to select the next potential APs to which the STA is likely to connect for context transfer. The SPCC execution where the STA context information is transferred from the old AP to the newly selected AP is also described.

In the normal active scanning procedure, the STA scans each channel in sequence. In SPCC, the normal active scanning procedure is modified to allow old AP to determine the right timing to transfer the STA's context. Moreover, the handoff is triggered when the RSSI of a new AP is at least above a predefined threshold for the associated AP [4]. Hysteresis is needed to avoid unnecessary handoff operations due to ping-pong effect where an STA is equally well served by different APs. In addition, the channel scanning sequence is changed so that the currently associated AP channel will be scanned last. Figure 2 shows the pseudo code for the active scanning procedure.

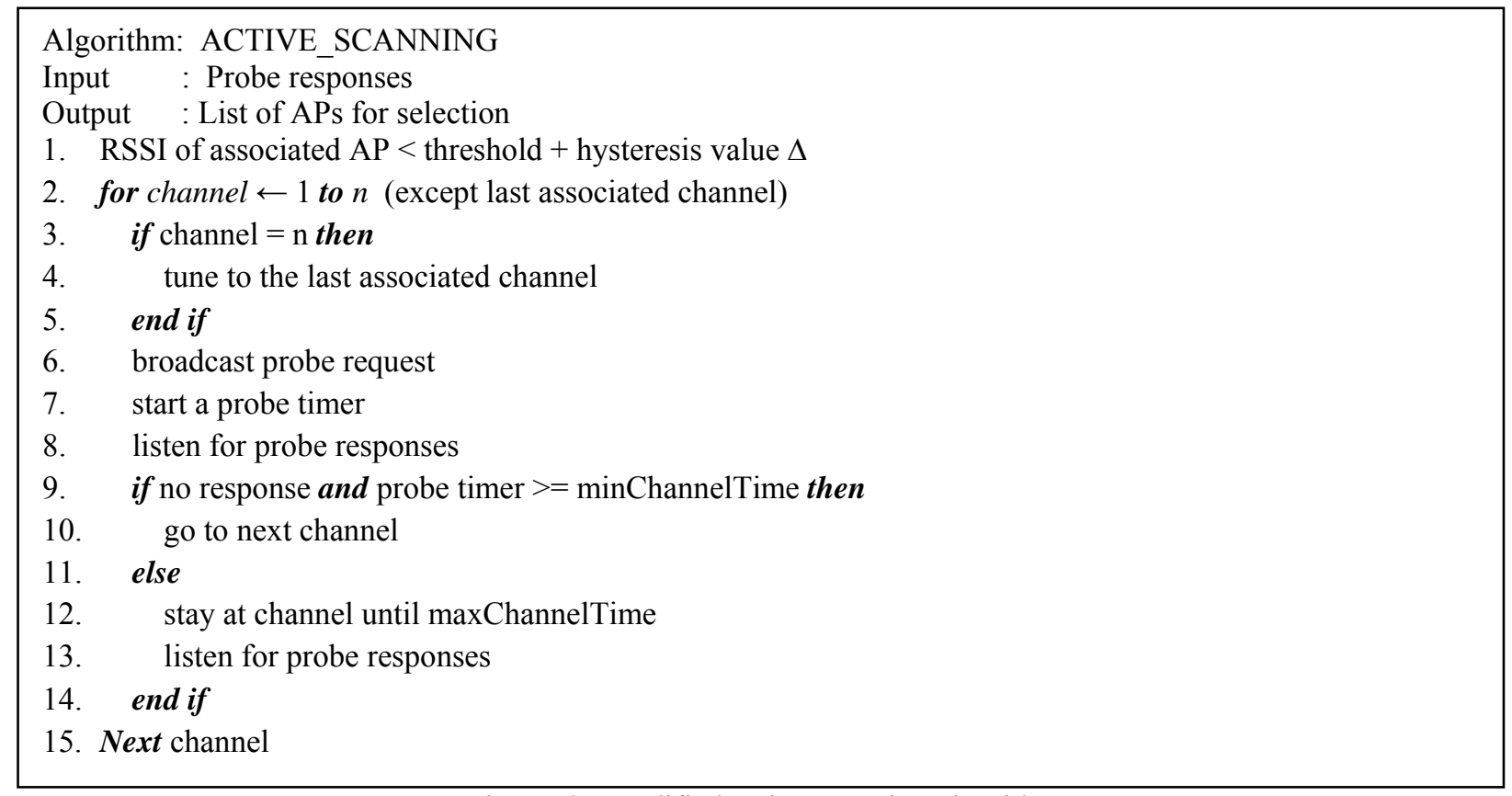

Fig 2: The modified active scanning algorithm

One of the most important considerations in SPCC is the selection of the next potential APs to which the STA context information will be transferred. When the neighboring APs receive a probe request frame from an STA, they send a link information packet to the old AP by attaching information such as the MAC address of AP, the MAC address of the STA and the RSSI of AP-STA via the high speed Ethernet LAN. The next potential AP determines the old AP from the modified probe request frame. On receiving the link quality packets from neighboring APs, the old AP first checks the existence of the information in its cache memory. If the information exists, it is refreshed. Otherwise, the AP stores the link information into its LinkInfoList in the cache memory. Hence, a list of potential neighboring APs to which the STA is likely to connect next is dynamically generated. Figure 3 shows the next potential AP selection algorithm. Only the related context information is sent to these potentials APs. 
The execution of SPCC involves three phases: (1) proactive context transfer, (2) processing of IAPP packets and (3) context removal. The purpose of proactive context transfer is to transfer STA context to next potential APs before the actual handoff is triggered. Based on the potential next APs selection algorithm, the old AP transfers the specific STA context to a set of potential APs stored in its cache. When the old AP receives a probe request frame from its associated STA (currently performing scanning process), the old AP looks for the AP-STA link information in its cache (LinkInfoList). If the link information exists, it transfers the STA context (Cache-Notify packets) to the listed APs via the high speed Ethernet LAN. Figure 4 shows the proactive context transfer algorithm.

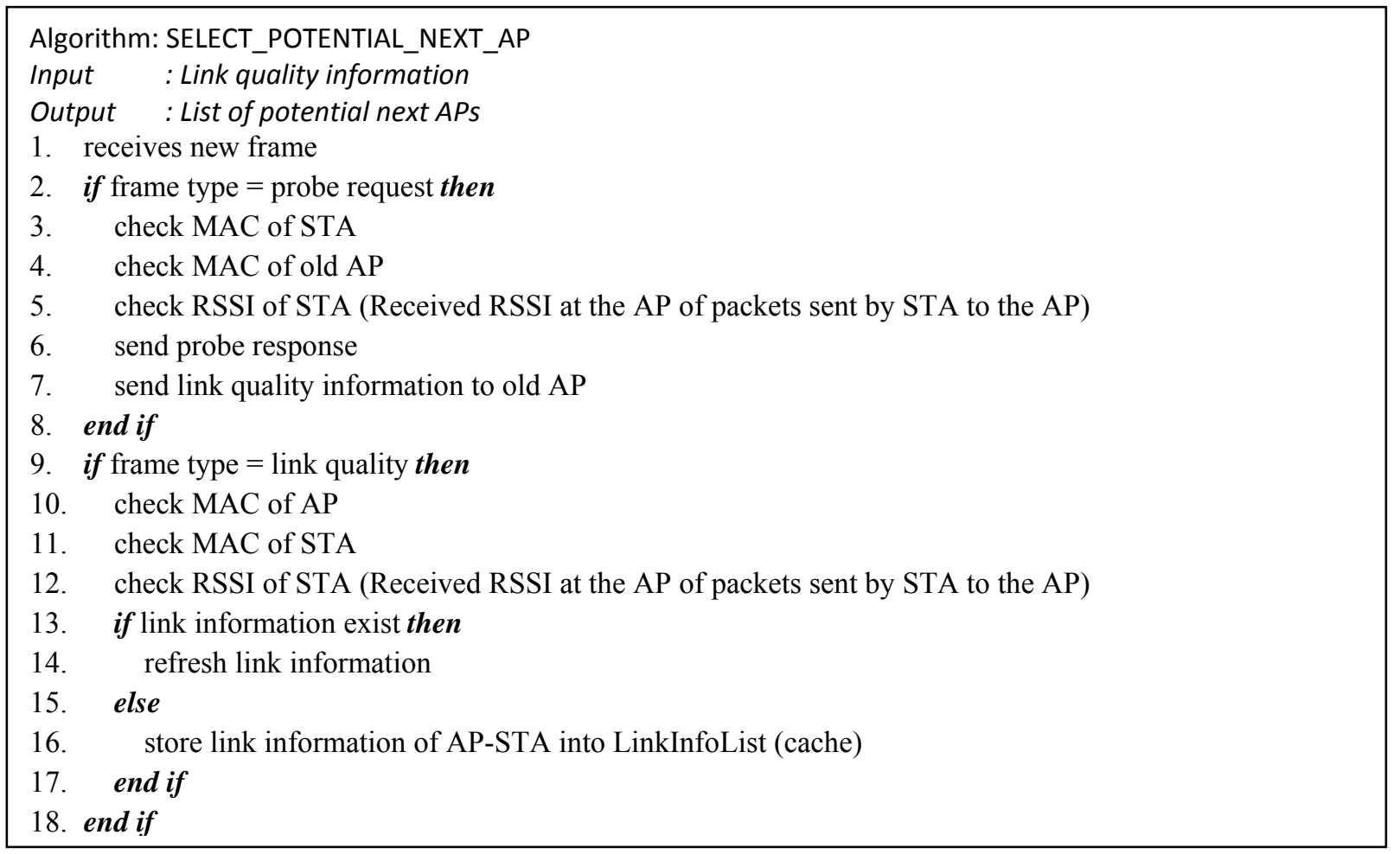

Fig 3: The next potential AP selection algorithm

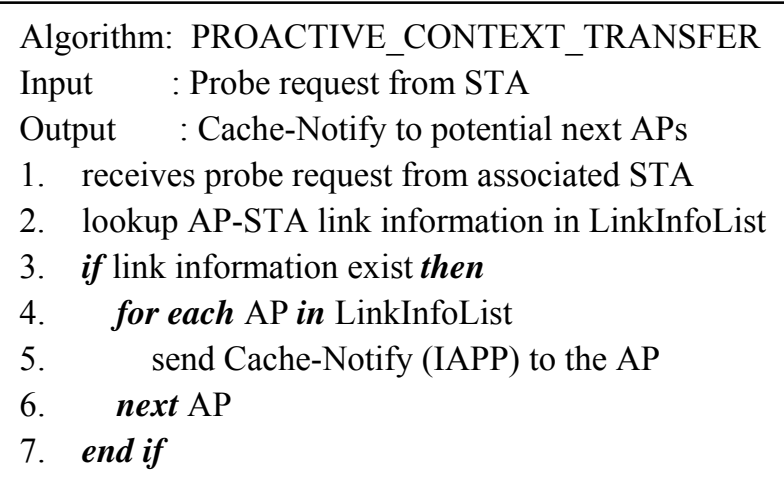

Fig 4: The algorithm to proactively transfer context to potential next APs 


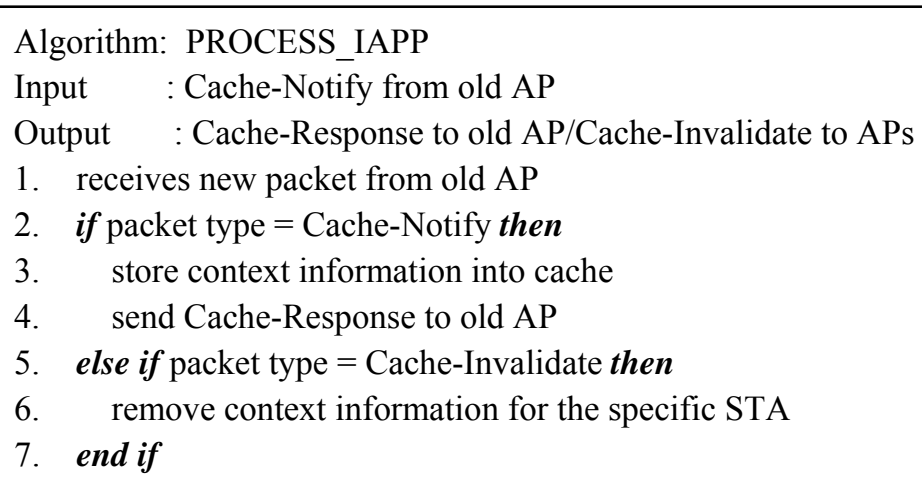

Fig 5: The algorithm to process IAPP packets

In our work, the IAPP is incorporated into SPCC. The context information regarding an STA-AP connection includes the STA MAC address, the STA association ID, old AP MAC address, the STA data frame sequence number, the old AP data frame sequence number and buffered data frame for the STA [12]. After the STA context information (Cache-Notify) is transferred to the potential next APs, these APs send Cache-Response packet back to the old AP to acknowledge the receipt of Cache-Notify. The Cache-Response packet carries the status of context transfer such as successful or stale cache. Figure 5 shows the IAPP packets processing algorithm. In addition, the Cache-Invalidate packets [8] are used to remove the context information for a specific STA from AP's cache. When the neighboring APs receive the Cache-Invalidate packets, the context information for the particular STA will be removed to reduce memory usage. To reduce the cache memory usage on an AP, a context removal algorithm is proposed. Figure 6 presents the context removal algorithm. After an AP accepts the re-association request from an STA; it sends Cache-Invalidate packets to the APs that receive the context information previously in order to remove the STA context information. The list of APs is based on the modified Cache-Notify packet which carries MAC addresses of the potential next APs.

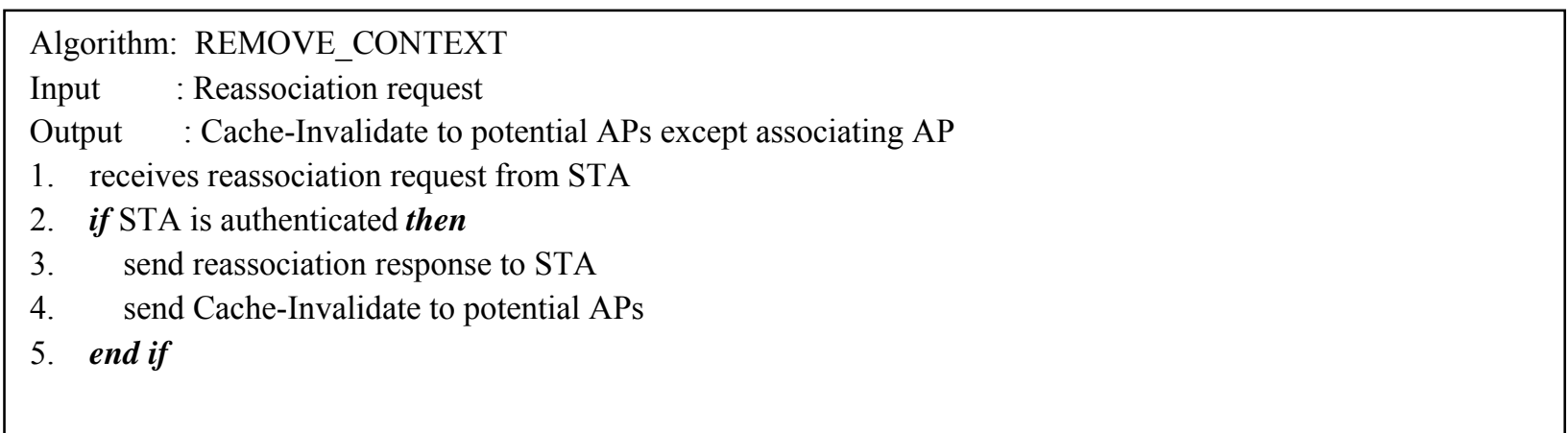

Fig 6: The algorithm to remove context information from related APs

\section{SIMULATION RESULTS AND DISCUSSION}

We present the simulation conducted in the OMNet++ environment to evaluate the proposed SPCC handoff mechanism. Section 4.1 describes the simulation plan, Section 4.2 presents the performance metrics and Section 4.3 demonstrates the simulation results.

\subsection{Simulation Plan and Environment}




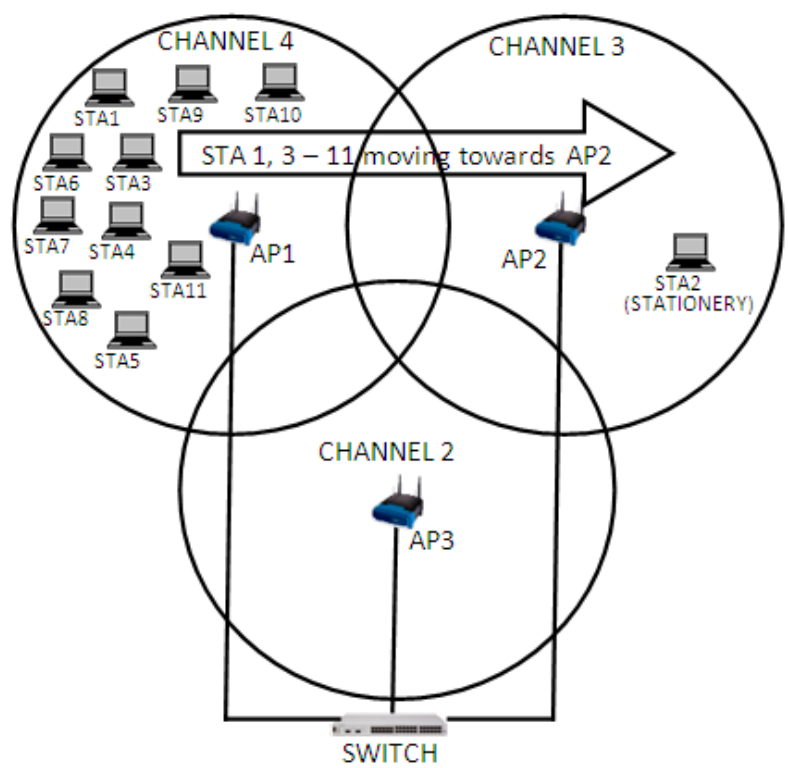

Fig 7: The simulation environment

The simulation environment uses a wireless network topology which consists of three APs, one switch, and a predefined number of STAs. All three APs are operating on different channels to avoid interference. The channel coverage of AP1, AP2 and AP3 are overlapped to allow for the continuous connectivity to the wireless network. Figure 7 shows the simulation environment where it consists of eleven STAs. Ten STAs are in AP1's radio range while one STA is in AP2's range. During the simulation, a predefined number of STAs move from AP1 towards AP2 and initiate handoff using the proposed SPCC mechanism and the standard IEEE 802.11 handoff mechanism (with IAPP). The presence of AP3 is to simulate an actual APs distribution whereby the STAs could moved into the region of AP3 as they move towards AP2. During this process, STA2 sends ping packets continually to all the predefined number of moving STAs. At the end of the simulation runtime, the re-association delay, ping packet dropped ratio and the effect of increased number of the STAs are inspected and compared.

\subsection{Performance Metrics}

Two metrics are defined to evaluate the performance of SPCC as compared to the standard handoff mechanism (with IAPP).

i. Re-association delay: This delay provides a direct insight into the amount of delay reduced by the SPCC mechanism during handoff.

ii. Ping packet dropped ratio: This parameter represents the ratio of total ping packets dropped over total packets send by the STA.

\subsection{Simulation Results (Scenario 2)}

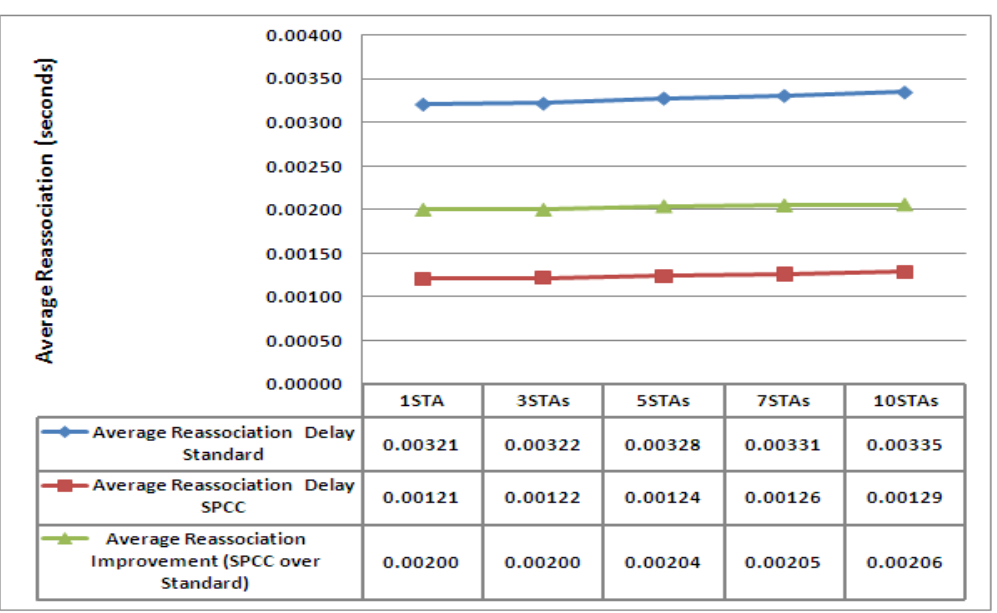

Fig 8: Average re-association delay comparison 
Figure 8 compares the average re-association delay for one STA, three STAs, five STAs, seven STAs and 10 STAs running on the SPCC mechanism and the standard handoff mechanism (with IAPP). The simulation results show that the average re-association delay increased slightly (both standard handoff and SPCC) when the number of STAs increases. This phenomenon is due to the contention in accessing the shared wireless medium during handoff. Despite the trend of increase in the average re-association delay, the SPCC mechanism recorded a lower reassociation delay compared to the standard handoff mechanism even when the number of STAs joining the network increases.

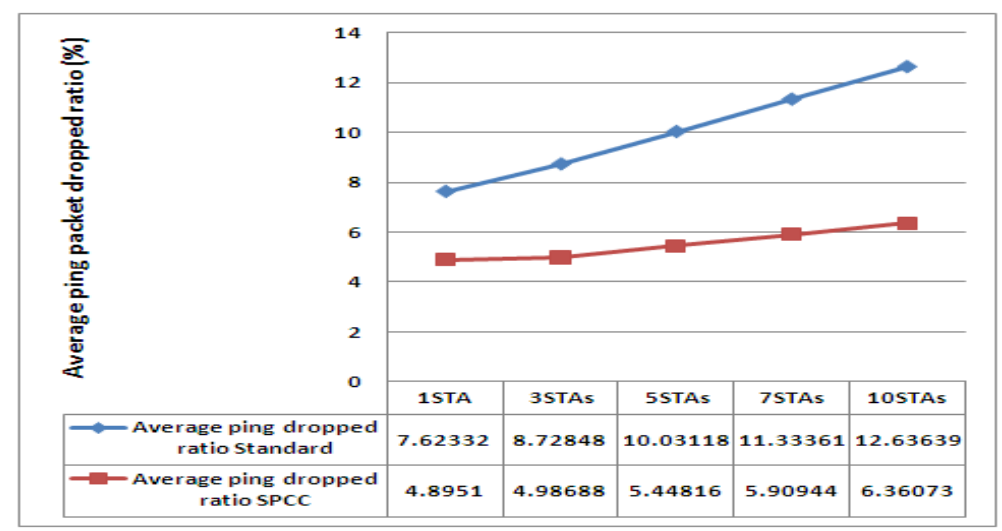

Fig 9: Average ping-packet dropped ratio

Figure 9 compares the ping packet dropped ratio while Figure 10 compares the average interruption time for handoff when the number of STAs increases. As shown in both the figures, the SPCC handoff mechanism outperforms the standard handoff mechanism for all the predefined numbers of STAs used in the simulation. Both mechanisms show an increase in the average ping packet dropped ratio and interruption time. This scenario can be explained as follows. As the number of STA increases, so does the possibility of the STAs transmitting data. Since IEEE 802.11 applies CSMA/CA in the shared medium, collision is more likely to happen. When collision is detected, all the STAs will invoke a backoff algorithm in which all the STAs will stop transmitting for a random amount of time. This causes a higher interruption time and directly affects the ping packet dropped ratio for both the handoff mechanisms.

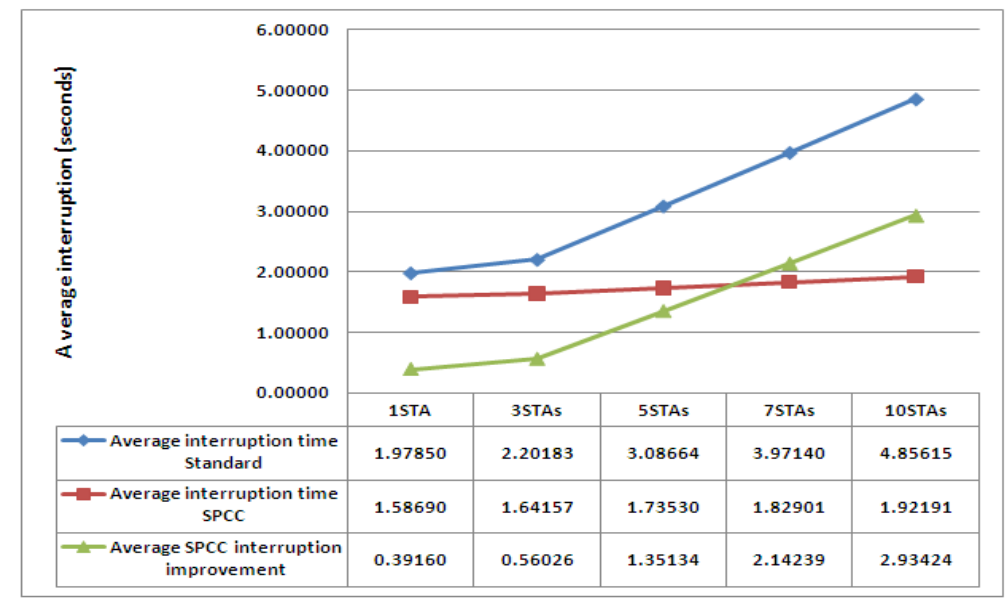

Fig 10: Average interruption time

\section{CONCLUSION}

In this paper, we have introduced a handoff mechanism, namely SPCC, which captures the next potential APs for context transfer in order to reduce the re-association delay. In our approach, the AP-STA link quality information is exchanged to determine the list of next potential APs. The existing IAPP procedure is modified and incorporated into the SPCC algorithm. We have implemented and evaluated the performance of the SPCC mechanism using OMNeT++ simulator equipped with the INET Framework. 
As compared to the regular handoff scheme, the performance of the proposed SPCC handoff mechanism shows significant improvement in terms of reducing the re-association latency. The SPCC mechanism provides $62 \%$ improvement by decreasing the re-association latency from $3.2 \mathrm{~ms}$ (regular handoff scheme) to $1.2 \mathrm{~ms}$ (SPCC). The SPCC handoff mechanism successfully reduces the service interruption time and at the same time also reduces the packet dropped ratio. The STA power-save mode is not implemented in this work. Therefore, an even lower or zero packet loss ratio is expected if the STA operates in the power save mode during handoff [10]; AP shall buffer the STA's MSDUs and only transmit them when the STA is back to active mode.

As previously discussed, the old AP forwards the STA's context information to a list of next potential APs during handoff. The overhead of transferring the context information is high when the number of APs visited by the STA increases during the scanning stage. Future work will consider predicting the AP with highest possibility to which the STA is most likely to connect next based on the AP-STA link quality information.

\section{REFERENCES}

[1] IEEE, "Part 11: Wireless LAN Medium Access Control (MAC) and Physical Layer (PHY) Specifications", IEEE Standard 802.11, 1999

[2] Pack, S \& Choi, Y, "Fast Inter-AP Handoff Using Predictive Authentication Scheme in a Public Wireless LAN Networks", The Proceedings of the Joint International Conference on Wireless LANs and Home Networks (ICWLHN 2002) and Networking (ICN 2002), Atlanta, USA.

[3] Sangheon, P \& Yanghee, C, "Pre-Authenticated Fast Handoff in a Public Wireless LAN Based on IEEE 802.1x Model", Proceedings of the IFIP TC6/WG6.8 Working Conference on Personal Wireless Communications, vol. 234. Kluwer B.V., Deventer, The Netherlands, 175-182, 2002

[4] Qing-An, Z \& Dharma, PA, "Handoff in wireless mobile networks", in Handbook of wireless networks and mobile computing, John Wiley \& Sons, Inc., pp. 1-25, 2002.

[5] IEEE, "Recommended Practice for Multi-Vendor Access Point Interoperability via an Inter-Access Point Protocol Across Distribution Systems Supporting IEEE 802.11 Operation”, IEEE 802.11f, 2003.

[6] Arunesh, M, Minho, S \& William, A, “An empirical analysis of the IEEE 802.11 MAC layer handoff process", SIGCOMM Computer Communication Review, vol. 33, no. 2, pp. 93-102, 2003.

[7] Kim, HS, Park, SH, Park, CS, Kim, JW \& Ko, SJ, "Selective Channel Scanning for Fast Handoff in Wireless LAN using Neighbour Graph", The 2004 International Technical Conference on Circuits/Systems Computers and Communications (ITC-CSCC2004) Japan, 2004.

[8] Mishra, A, Shin, M \& Arbaush, WA, "Context caching using neighbour graphs for fast handoffs in a wireless network", INFOCOM 2004. Twenty-third Annual Joint Conference of the IEEE Computer and Communications Societies, p. 361, 2004.

[9] IEEE, "802.1X Port-Based Network Access Control”, IEEE Standard for Local and metropolitan area networks, 2004.

[10] Ramani, I \& Savage, S, "SyncScan: practical fast handoff for 802.11 infrastructure networks", INFOCOM 2005. 24th Annual Joint Conference of the IEEE Computer and Communications Societies. Proceedings IEEE, pp. 675-84, 2005.

[11] Vladimir, B, Arunesh, M \& Suman, B, "Eliminating handoff latencies in 802.11 WLANs using multiple radios: applications, experience, and evaluation", Proceedings of the Internet Measurement Conference 2005 on Internet Measurement Conference, Berkeley, CA, pp. 27-27, 2005.

[12] Wang, J \& Bao, L, "Mobile Context Handoff in Distributed IEEE 802.11 Systems", International Conference on Wireless Networks, Communications and Mobile Computing, pp. 680- 5, 2005.

[13] Loughney, J, Nakhjiri, M, Perkins, C \& Koodli, R, Context Transfer Protocol RFC4067, 2005.

[14] Aura, T \& Roe, M, "Reducing Re-authentication Delay in Wireless Networks", First International Conference on Security and Privacy for Emerging Areas in Communications Networks, 2005. SecureComm 2005. , pp. $139-48,2005$

[15] Ha, D, Arek, D \& Steven, G (2005), 'Proactive context transfer and forced handover in IEEE 802.11 wireless LAN based access networks', SIGMOBILE Mobile Computing Communication Review, vol. 9, no. 3, pp. 32-44.

[16] Ramachandran, K, Rangarajan, S \& Lin, JC, "Make-Before-Break MAC Layer Handoff in 802.11 Wireless Networks”, IEEE International Conference on Communications, 2006. ICC '06. , pp. 4818-23, 2006.

[17] Vivek, M \& Konstantina, P, "Using smart triggers for improved user performance in 802.11 wireless networks", Proceedings of the 4th international conference on Mobile systems, applications and services, Uppsala, Sweden, 2006

[18] Jaeouk, O, Morales, P, Darmawan, A \& Morikawa, HAMH, "Using Shared Beacon Channel for Fast Handoff in IEEE 802.11 Wireless Networks", IEEE 65th Vehicular Technology Conference, 2007. VTC2007-Spring., pp. $849-53,2007$ 
[19] Wu, H, Tan, K, Zhang, Y \& Zhang, QAZQ, "Proactive Scan: Fast Handoff with Smart Triggers for 802.11 Wireless LAN", IEEE INFOCOM 2007. 26th IEEE International Conference on Computer Communications, pp. 749-57, 2007.

[20] Varga, A, OMNeT++ Discrete Event Simulation System Version 3.3, OMNeT++ (OMNEST), viewed 1 December 2008, http://www.omnetpp.org.

[21] Pack, S et al., "SNC: A Selective Neighbor Caching Scheme for Handoff in IEEE802.11 Wireless Networks", ACM SIGMOBILE Mobile Computing and Communication Review, 9(4),pp 39-49, October 2005. 